interested in further information about $\mathrm{HNS} / \mathrm{nES}$, 2) if they would be willing to try HNS/nES, and 3) if they were to choose only one of the four listed treatments, which one would they prefer to use every night.

Results 162 patients completed the survey ( 81 males, mean age 52 (12) years, BMI 34 (7.3) kg/m², ESS 10.2 (6.0) points, FOSQ10 28.5 (8.1) points). The majority of the respondents $(89.5 \%)$ had been diagnosed with OSA, with $95.4 \%$ of those being treated with CPAP. $91.3 \%$ of the respondents were interested in more information and were willing to try HNS/nES. Most respondents preferred the potential use of nES (56.7\%), while $21.7 \%$ chose HNS, $17.8 \%$ CPAP, and 3.8\% the MAD. There were no differences in the characteristics of the patients who preferred nES compared to those who preferred other treatments; however, a regression analysis showed that a low ESS score was a predictor of patients choosing nES $(p<0.05)$. Conclusion Although the CPAP is the established treatment for OSA, most patients would prefer alternatives for long-term treatment. The majority of the respondents were interested in emerging technologies, with less sleepy patients more likely to choose less invasive treatment options.

\section{P296 EFFECTIVENESS OF ADAPTIVE SERVO VENTILATION IN THE TREATMENT OF CENTRAL SLEEP APNEA}

${ }^{1}$ Ali Al-Ameri, ${ }^{1}$ Martin Latham, ${ }^{2}$ Jayne Pateraki, 'Mark Elliott. 'St James's University Hospital, Leeds, UK; ${ }^{2}$ York Teaching Hospital, York, UK

\subsection{6/thoraxinl-2014-206260.414}

Background Adaptive Servo Ventilation (ASV) was developed to treat Central Sleep Apnea in patients with heart failure, which is usually associated with a low or normal PaCO2. The aim of ASV is to stabilise rather than increase overall ventilation. Evidence is limited regarding the use of ASV not only in heart failure patients but central sleep apnea of other aetiologies. The current study therefore explored this therapy in a regional sleep centre in the UK.

Method A retrospective review of the outcomes of 42 patients who were treated with ASV between January 2012 and December 2013, either following conventional positive airway pressure (PAP) or as an initial therapy. Measurements included the Apnea Hypopnea Index (AHI), compliance (measured by hours of machine use/night) and subjective sleep quality, pre and post ASV.

Results All patients demonstrated evidence of central sleep apnea with a reduced or normal transcutaneous $\mathrm{CO} 2$ during daytime spontaneous ventilation. Seven patients (16\%) met the criteria for complex sleep apnea. 16 (38\%) had evidence of heart

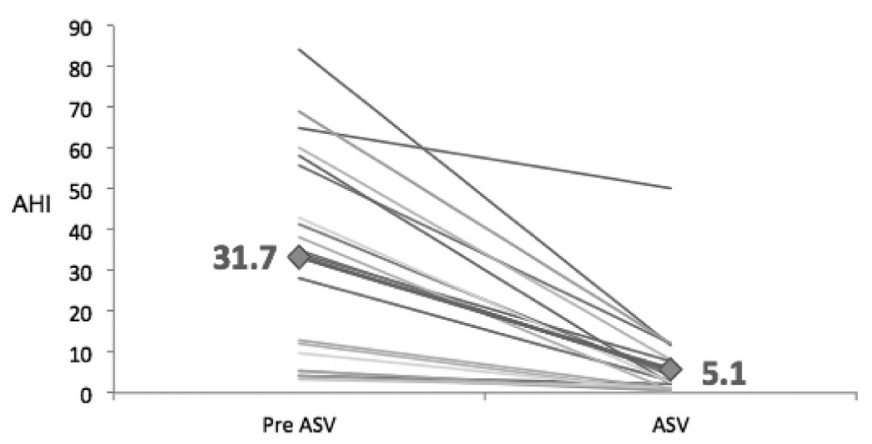

Abstract P296 Figure 1 AHI pre and post the use of ASV failure whilst opioids were in use in six patients (14\%). The majority of patients, $(\mathrm{n}=36,86 \%)$, were on PAP prior to ASV (mean duration 2.4 years), 22 patients (53\%) were on Bi-level and $14(33 \%)$ were on CPAP. Six patients (14\%) had ASV as an initial therapy. The mean AHI improved from 31.7/h (range 2$84 / \mathrm{h}$ ) to $5.1 / \mathrm{h}$ (Range $0-50 / \mathrm{h}$ ) with ASV [Figure 1]. Compliance improved from $5.2 \mathrm{~h} /$ night to $6.4 \mathrm{~h} /$ night with ASV. 22 patients $(52 \%)$ reported a subjective improvement in their sleep quality using ASV.

Conclusion ASV appeared superior to traditional PAP in improving AHI, compliance and sleep quality for patients with central sleep apnea of various aetiologies.

\section{From hospital to home: NIV in clinical practice}

\section{P297 EFFECT OF BTS-RECOMMENDED MEDICAL LEADERSHIP ON THE "DOOR-TO-MASK" TIME OF ACUTE NON- INVASIVE VENTILATION (NIV) SET UPS}

${ }^{1} \mathrm{H}$ Boryslawskyj, ${ }^{2} \mathrm{~F}$ Rauf, ${ }^{2} \mathrm{~B}$ Beauchamp, ${ }^{2} \mathrm{~A}$ Oakes, ${ }^{2} \mathrm{~N}$ Santana-Vaz, ${ }^{3} \mathrm{~B}$ Chakraborty, ${ }^{2} \mathrm{R}$ Mukherjee. 'School of Clinical and Experimental Medicine, University of Birmingham, Birmingham, UK; ${ }^{2}$ Birmingham Heartlands Hospital, Birmingham, UK; ${ }^{3}$ School of Mathematics, University of Birmingham, Birmingham, UK

\subsection{6/thoraxjnl-2014-206260.415}

Introduction NIV is now part of standard acute care in the UK. "Door-to-mask" time has been discussed as a performance/quality indicator of acute NIV services [Mandal S et al. Thorax, 66(4). A117]. We compare the "Door-to-mask" time by analysing the "\% of patients receiving NIV within 3 h" of diagnosis of acute hypercapnic respiratory failure (AHRF) at two acute hospitals in central England: Hospital A, which appointed a Lead NIV consultant in 2009-10 as per BTS recommendations and Hospital B without a Lead consultant. Both hospitals are run by the same Trust and on call physiotherapy teams, with comparable acute catchment sizes.

Methods The survey was approved as an audit by the Trust's Clinical Standards Committee. Data was taken from the acute NIV database, maintained continuously since 2004 at HospitalA and since 2009 Hospital B as part of a drive to maintain built-in quality. All acute NIV episodes between 01/10/2010-01/04/2011 (period 1) and 01/10/2012-01/04/2013 (period 2) were included: 458 episodes (27 excluded - incomplete data).

Results In period1, the " $\%$ of patients receiving NIV within 3 h" of diagnosis of AHRF were 69.9\% at Hospital A and 69.49\% at Hospital B. In period2, Hospital A improved to $82 \%$ with Hospital B at $71.1 \%$. The most significant improvement, however, was in the reduction of variance around the median "Doorto-mask" time of $1.55 \mathrm{~h}$ at Hospital A and $1.83 \mathrm{~h}$ at HospitalB on the Probability Density curves, also seen over other periods outside the ones studied.

Conclusions The service at Hospital B did not show any measurable improvement in 'door-to-mask time' between periods1 and 2 but Hospital A did. As there were no significant differences like the demography, work load, frequency of on calls or number/grades of staff between the periods 1 and 2, this improvement could be a reflection on the role of a Lead NIV consultant at Hospital A as per BTS recommendations. Furthermore, reduction of variance around the median "Door-to-mask" time is observed to be a consistent feature of the improvement, which 
needs evaluation as an independent performance/quality indicator of acute NIV services.

\section{P298 REFERRAL PATTERNS AND MORTALITY IN A NON- INVASIVE VENTILATION (NIV) UNIT IN A TERTIARY UNIVERSITY HOSPITAL IN THE UK}

K Aldridge, S Bikmalla, A Thomas. University Hospital of North Staffordshire, Stoke-onTrent, UK

\subsection{6/thoraxjnl-2014-206260.416}

Introduction NIV for acute hypercapnic respiratory failure (AHRF) in COPD and restrictive lung disease has become widespread in the UK. Early institution of NIV in appropriate patients gives the best outcome.

Methods We retrospectively examined referral patterns to our tertiary 12 bedded NIV unit during a 12 month period from November 2012 to October 2013. Admission criteria to the unit is standardised and through the NIV consultant or senior nursing staff. Site of referral was noted and mortality rate was calculated.

Results 612 referrals were made to the dedicated NIV unit in the 12 months. 125 were elective admissions for setting up domiciliary NIV and were excluded from the mortality analysis as there was no mortality in this group. The overall mortality for the rest of the cohort was $15.2 \%$ of the remaining 487 patients acutely admitted to the unit. The source of referrals to the unit was varied and as shown in Figure 1. The mortality rate for admissions from the acute portals ( $\mathrm{A}$ and $\mathrm{E}$ and the Acute Medical unit) were significantly lower (10.4\%) than from the medical wards (23\%). This reflects the fact that even with a well selected cohort the timing of the respiratory failure in the course of illness plays an important part in determining mortality. We know that uncorrected respiratory acidosis after $4 \mathrm{~h}$ of NIV is a strong determinant of mortality. The highest mortality (64.5\%) was seen of referrals from the Frail elderly unit. This also shows that general constitution plays an important role in mortality.

Conclusion Early referral for NIV support in AHRF improves outcomes. Delayed hypercapnic respiratory failure and frailty are important factors determining poorer outcomes.

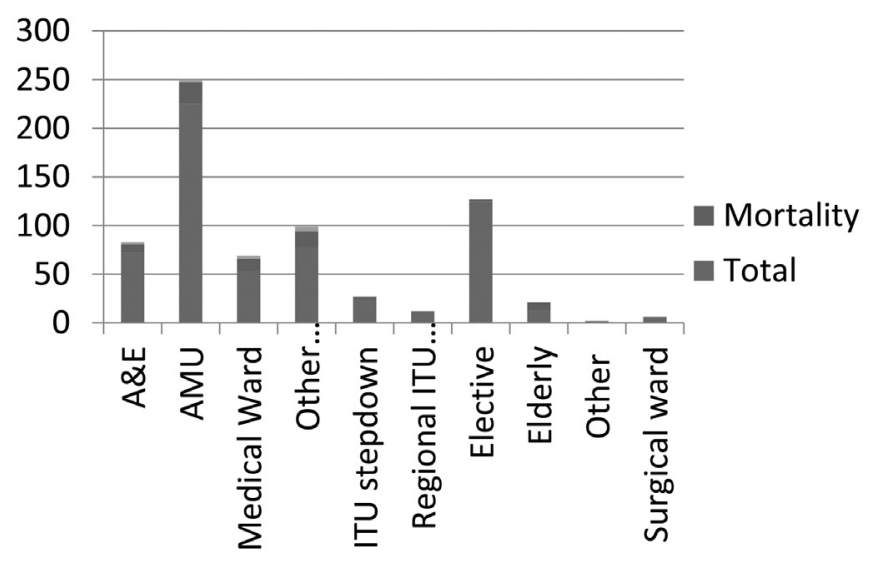

Abstract P298 Figure 1
P299 THE ROLE OF A MULTIDISCIPLINARY RESPIRATORY HUB IN IMPROVING POST-DISCHARGE FOLLOW UP OF PATIENTS RECEIVING ACUTE NON-INVASIVE VENTILATION (NIV)

${ }^{1} \mathrm{~F}$ Rauf, ${ }^{1} \mathrm{~A}$ Oakes, ${ }^{1} \mathrm{Y}$ Khan, ${ }^{1} \mathrm{~T}$ Stuart, ${ }^{2} \mathrm{~B}$ Chakraborty, ${ }^{3} \mathrm{AM}$ Turner, ${ }^{1} \mathrm{R}$ Mukherjee. ${ }^{1}$ Birmingham Heartlands Hospital, Birmingham, UK; ${ }^{2}$ School of Mathematics, University of Birmingham, Birmingham, UK; ${ }^{3}$ College of Medical and Dental Sciences, University of Birmingham, Birmingham, UK

10.1136/thoraxjnl-2014-206260.417

Introduction Post-discharge follow up rate is a national audit metric for acute NIV services in the UK [Davies M. Adult NIV Audit report. BTS Reports 2012. 4 (3): 9-10.]. Appropriate respiratory follow up reduces the number of re-admissions [Turner AM et al. Prim Care Respir J 2013; 22(1):72-78.] In order to improve post-discharge follow ups, a rapid access one-stop multidisciplinary respiratory outpatient Hub was created at our 709bedded acute hospital in October 2011.

Methods From the acute NIV database maintained continuously since 2004 , we analysed the proportion of discharges that were offered respiratory follow up within 6 months of discharge after Acute NIV during the calendar year 2009 (pre-Hub) and first 6 months of 2013 (post-Hub). Chi-squared test was performed for statistical significance of the observed differences.

Results The proportion of unique discharges offered a follow up appointment at the time of discharge improved from 57\% (62/ 107) pre-Hub to $80 \%(36 / 45)$ post-Hub: $\mathrm{p}=0.009$. The proportion of patients attending follow-up appointments increased from $40 \%(42 / 107)$ pre-Hub to $58 \%$ (26/45) post-Hub: $\mathrm{p}=$ 0.036 , confirming a statistically significant improvement. The number of acute NIV re-admissions dropped between 2009 and 2013 but expectedly not statistically significant, as only 6 months' data from the post-Hub period was analysed against 12 months of data from the pre-Hub period.

Conclusions The increase in the number of patients attending post-discharge NIV follow up correlates with the direct increase in the number being offered follow up, an improvement most obviously measuring the impact of the multidisciplinary 'Hub'. The Hub would also be the most plausible explanation for the drop in acute NIV re-admissions between the 2 periods, not the least because apart from a 'routine' follow-up, it supports community teams and provides an alternative to ambulance calls to people with complex respiratory needs in a responsive fashion. Further longitudinal evaluation of the Multidisciplinary Hub is necessary to fully understand its impact on the quality and safety of complex respiratory care.

\section{P300 THE CLINICAL EFFECTIVENESS OF DOMICILIARY NON- INVASIVE VENTILATION (NIV) IN PATIENTS WITH END- STAGE COPD}

${ }^{1}$ J Dretzke, ${ }^{2} \mathrm{C}$ Dave, ${ }^{1} \mathrm{D}$ Blissett, ${ }^{2} \mathrm{R}$ Mukheriee, ${ }^{1} \mathrm{M}$ Price, ${ }^{1} \mathrm{~S}$ Bayliss, ${ }^{1} \mathrm{X} W u$, ${ }^{1} \mathrm{R}$ Jordan, ${ }^{1} \mathrm{~S}$ Jowett, 'DJ Moore, 'AM Turner. 'University of Birmingham, Birmingham, UK; ${ }^{1}$ Heart of England NHS Foundation Trust, Birmingham, UK

10.1136/thoraxjnl-2014-206260.418

Background NIV is very effective when used acutely in hospital during acute exacerbations of COPD, however, evidence supporting its use in a home setting for more stable COPD patients is limited. In the UK domiciliary NIV is considered by many 